\title{
Geißler, Gert
}

\section{Friedrich Adolph Wilhelm Diesterweg}

formal und inhaltlich überarbeitete Version der Originalveröffentlichung in: formally and content revised edition of the original source in:

Jugendhilfe 53 (2015) 4, S. 260-264

Bitte verwenden Sie in der Quellenangabe folgende URN oder DOI /

Please use the following URN or DOI for reference:

urn:nbn:de:0111-pedocs-152580

10.25656/01:15258

https://nbn-resolving.org/urn:nbn:de:0111-pedocs-152580

https://doi.org/10.25656/01:15258

\section{Nutzungsbedingungen}

Gewährt wird ein nicht exklusives, nicht übertragbares, persönliches und beschränktes Recht auf Nutzung dieses Dokuments. Dieses Dokument is ausschließlich für den persönlichen, nicht-kommerziellen Gebrauch bestimmt. Die Nutzung stellt keine Übertragung des Eigentumsrechts an diesem Dokument dar und gilt vorbehaltlich der folgenden Einschränkungen: Auf sämtlichen Kopien dieses Dokuments müssen alle Urheberrechtshinweise und sonstigen Hinweise auf gesetzlichen Schutz beibehalten werden. Sie dürfen dieses Dokument nicht in irgendeiner Weise abändern, noch dürfen Sie dieses Dokument für öffentliche oder kommerzielle Zwecke vervielfältigen, öffentlich ausstellen, aufführen, vertreiben oder anderweitig nutzen.

Mit der Verwendung dieses Dokuments erkennen Sie die Nutzungsbedingungen an.

\section{Terms of use}

We grant a non-exclusive, non-transferable, individual and limited right to using this document

This document is solely intended for your personal, non-commercial use. Use of this document does not include any transfer of property rights and it is conditional to the following limitations: All of the copies of this documents must retain all copyright information and other information regarding legal protection. You are not allowed to alter this document in any way, to copy it for public or commercial purposes, to exhibit the document in public, to perform, distribute or otherwise use the document in public.

By using this particular document, you accept the above-stated conditions of use.

\section{Kontakt / Contact:}

\section{peDOCS}

DIPF | Leibniz-Institut für Bildungsforschung und Bildungsinformation Informationszentrum (IZ) Bildung

E-Mail: pedocs@dipf.de

Internet: www.pedocs.de

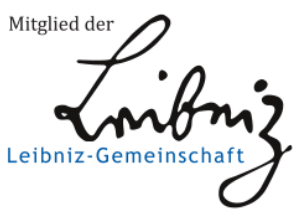




\section{Friedrich Adolph Wilhelm Diesterweg (1790-1866)}

\section{Leben und Werk}

Geboren am 29. Oktober 1790 in Siegen als siebtes Kind einer dort alt eingesessenen Familie, hat Diesterweg die Geschichte des pädagogischen Denkens, der Schule und der Lehrerschaft in Deutschland im 19. Jahrhundert unverwechselbar beeinflusst. Er wurde Zeuge rasanter Entwicklungen in Naturwissenschaft und Technik, von tiefgehenden Wandlungen der Gesellschaft. Als Direktor des Elementarschullehrer-Seminars in Moers seit 1820, vor allem aber als Leiter der Seminars für Stadtschullehrer in Berlin ab 1832 war konfrontiert mit den sozialen Gegensätzen der aufkommenden Industriegesellschaft und zugleich hineingestellt in Auseinandersetzungen um den Gewinn von bürgerlichen Freiheitsrechten.

Von daher richtete sich sein ganzes Bemühen darauf, die in seiner Zeit liegenden Möglichkeiten individueller und gemeinschaftlicher Entwicklung der Menschen gegen ständische Prinzipien durchzusetzen und sie in der Gestaltung von Erziehung, Schulunterricht und Schulwesen fassbar zu machen. Seine Wirkung beruhte auf dem Zusammenhang von theoretischem Schaffen, praktischer Tätigkeit, politischem Engagement und persönlicher Haltung. Damit konnte er vor und in der Revolution von 1848 zu einer Symbolfigur für den selbstbewussten, von Emanzipationsideen erfassten Teil der Volksschullehrer werden.

Zusehends gewann der Berliner Seminardirektor in Lehrerkreisen, die der Obrigkeit zu widerstreben begannen, an schulpolitischer und pädagogischer Autorität. Während sich andere Pädagogen und Schulmänner unter dem Druck der restaurativen politischen Verhältnisse auf unverfängliche Fachfragen zurückzogen, prägte Diesterweg, der Pädagogik durchaus auch „als einen Teil der Politik“ (SW 3; S. 59) verstehen konnte, in der Beschäftigung auch mit der sozialen Problematik der Zeit gesellschaftskritisches Denken aus. Mit seinen seit 1827 herausgegebenen Rheinischen Blättern trat er immer mehr in „systematische Opposition“ (SW 9; S. 51) zu den herrschenden politischen Bestrebungen.

Nach der Niederlage der Reformkräfte wurde Diesterweg im Juli 1850 vom zuständigen preußischen Ministerium in den Ruhestand versetzt. Nun ohne noch Rücksicht auf beamtenrechtliche Verpflichtungen nehmen zu müssen, entfaltete er in seiner Publizistik und seit 1858 als Mitglied des liberalen Flügels im preußischen Abgeordnetenhauses sein sich über weite Bereiche der Wissenschaften erstreckendes Wissen, den Reichtum seiner Sprache, sein Vermögen zu zwingender und eindringlicher Argumentation. Eben auch des Schulfortschritts wegen drang er auf die Erfüllung jener Artikel der preußischen Verfassung, die entgegen den tatsächlichen Maßnahmen rechtliche Voraussetzungen für eine Entwicklung zur Rechtsstaatlichkeit boten, so insbesondere die Artikel 4 (Gleichheit aller Staatsbürger in Rechten und Pflichten), 12 (Freiheit der religiösen Bekenntnisse), 26 (Unterrichtsgesetz), 27 (Recht auf freie Meinungsäußerung, Pressefreiheit, Abschaffung der Zensur), 29 (Versammlungsfreiheit), 30 (Recht zur Vereinigung in Gesellschaften).

Vier Tage nach Annahme seiner Wiederwahl zum Abgeordneten verstarb der Pädagoge am 7. Juli 1866 in Berlin.

Diesterwegs Schrifttum ist mit einer Fülle von Artikeln und Rezension vor allem in den von ihm 1827 begründeten und von ihm bis zum seinem Tode herausgegebenen „Rheinischen Blättern für Erziehung und Unterricht“" versammelt. Im Jahre 1835 entstand die erste Fas- 
sung seines später noch mehrfach aufgelegten und weithin rezipierten „Wegweisers zur Bildung für deutsche Lehrer". In diesem Werk, das in vierter Auflage 1850 in zwei Bänden schließlich 1544 Seiten umfasste und das moderne pädagogische Wissen der Zeit zusammenführte, legte er ein relativ geschlossenes System didaktischer Aussagen vor, in dem auf der Grundlage seines Menschenbildes, seines Erziehungszieles und des Gedankens der allgemeinen Menschenbildung wesentliche Erkenntnisse der vorangegangenen Pädagogik dergestalt zusammengefasst, verdichtet und geordnet sind, dass es als ein Handlungskonzept für den Volksschullehrer wirksam werden konnte. In Auseinandersetzungen mit dem herkömmlichen Unterricht, der einseitigen „Gedächtniskultur“, der „Wissensanhäufung“, also der „materialen“ Bildung, betonte er die „formale“ Seite des Unterrichts, den Gedanken der „Kräftebildung“" der geistigen Selbständigkeit.

Die reifsten Ergebnisse seines Nachdenkens finden sich in einer Reihe von Spätschriften, die sowohl in pädagogischer als auch in bildungspolitischer und sozialer Hinsicht programmatischen Charakter tragen. Für jeden Staatsbürger Freiheit der Entwicklung seiner Anlagen und Fähigkeiten, für jeden Staatsbürger Gleichheit der Bildungsmöglichkeiten, für jeden Staatsbürger gleiche Rechte und Pflichten, für jeden Staatsbürger eine gesicherte soziale Existenz, sind ihre wichtigsten Ansatzpunkte.

\section{Pädagogische Potenziale}

Die von ihm skeptisch beobachteten temporären Konjunkturen philosophischer Anschauungen bedeuteten für Diesterweg, von der üblichen Bindung der Pädagogik an ein bestimmtes, zumal ein zeitgenössisches philosophisches Lehrgebäude abzusehen. Je mehr zu seinen Lebzeiten konservatives Denken Geltung erlangte, umso stärker forderte er „Selbständigkeit der Pädagogik (die Anthropologie einschließend) als Wissenschaft" (SW 13; S. 209). Eine solche Pädagogik als Lehre systematisch auszufeilen, lag ihm fern. Der Pädagoge verfahre nicht nach den Diktaten eines Systems; er befolge „nur Grundsätze“ (SW 20; 376). Die Pädagogik müsse zugleich „eine feste und eine bewegliche Wissenschaft" (SW 13; S. 158). Sie müsse sich dynamisch entwickeln, zugleich von festen Prinzipien geleitet sein (SW 13; S. 108). Sie habe in diesem Sinne mit der Zeit zu gehen und ihren Bedürfnissen zu entsprechen.

Von daher hat Diesterweg seine Pädagogik in dieser der jener Dimension nicht so tief begründet und im Einzelnen ausgeführt wie der eine oder andere seiner Zeit- und Zunftgenossen. Ausgreifend wie kaum einer von diesen nahm er jedoch über philosophische Bezüge hinaus Kenntnis von allgemeinen, für Erziehung und Bildung wie auch immer bedeutsamem Erträgen der Wissenschaftsentwicklung. Insbesondere hat er bis hin zu den schulpädagogischen Konsequenzen das Erziehungsdenken den Naturwissenschaften und allen jenen Ideen und Beobachtungen geöffnet, die sozialen Fragen galten. Das hat seinen pädagogischen Entwürfen nicht ein Hochmaß an theoretischer Konzentration, sicher aber Gewinn an gesellschaftlicher Blickweite gebracht. Sein Erziehungsdenken läuft auf Anregungen, Anstöße, Impulse, nicht auf systemgebundenes Durchführen hinaus. Kennzeichnend für dieses ist die Bevorzugung von Induktion, Beobachtung und Empirie. Er schätzte das Konkrete, die Anschaulichkeit, jedoch nicht, um letztere zu verabsolutieren, sondern als Faktoren der Entwicklung des Allgemeinen durch „die analysierende Verstandestätigkeit“ (SW 3; S. 245) wirken zu lassen.

Als universales Prinzip der Menschenbildung bezeichnete Diesterweg die Selbsttätigkeit, und zwar „im Dienste des Wahren, Schönen und Guten“. Überzeugt davon, dass der Mensch grundsätzlich zum Guten disponiert sei, war ihm die Selbsttäti9gkeit der Schlüssel zur erstrebten Selbstbestimmung des Menschen. Nach diesem Prinzip sollten alle zu Menschen im 
„umfassenden, erhabenen und begeisternden Sinne des Wortes“ (SW 7; S. 89) erzogen werden. Die „freie Entwicklung der menschlichen Individualität" (SW 11; S. 202) stehe ihm obenan, jeder solle „,nach seiner Fähigkeit und Begabung..., wenn es sein kann, in den Himmel wachsen“ (SW 11; S. 204). Der Entwicklungsgedanke entsprach nach Diesterweg dem Grundsatz der Freiheit, insofern er verlange, dass jedem Menschen die Entwicklung seiner Fähigkeiten und seiner individuellen Möglichkeiten gestattet und durch gesellschaftliche Einrichtungen auch gesichert sein solle. Dazu trat das Prinzip der Gleichheit, das die Sicherung dieser Möglichkeiten durch die Gesellschaft für grundsätzlich alle Kinder gebot.

Nach Diesterweg musste Erziehung naturgemäß sein, denn: „Jeder Mensch gehört zu den Naturwesen, seinem Körper nach zu der äußeren, seinem Geiste nach zur innern Natur. Die Erscheinungen, Äußerungen, Tatsachen usw. beider Seiten der Menschennatur geschehen nach den ihnen einverleibten, ihnen immanenten Gesetzen" (SW 6; S. 445). Diese Naturvorgaben waren zu erkennen und zu beachten (SW 16; S. 331ff.). Grundsätzlich müsse der Menschenerzieher deshalb ,auf die Psychologie einen entscheidenden, hohen, absoluten Wert“ legen (SW 18; S. 289), das Wesen, die Anlagen und Fähigkeiten der zu Bildenden erfassen, überall „die Winke der Natur“ respektvoll verflogen und naturgemäß verfahren (SW 18, S 289).

Zunehmend hatte Diesterweg jedoch auch erfahren, dass die Menschen ihre Natur je in einer bestimmten ,äußeren Lage“ zu entfalten hatten, dass Erziehung durch soziale Faktoren gebunden war. Die Begegnung mit dem allgegenwärtigen Elend zuerst in den Fabrikgebieten des Rheinlandes, dann in Berlin, führte ihn zu der Feststellung, dass die Entwicklung der Menschen wesentlich, ja primär vom „Brot“, von ihren jeweiligen Lebensverhältnissen abhängig war. „Die Menschen werden, was sie werden können, das Resultat entspricht den Bedingungen, unter denen sie lebten, und den Kräften, die auf sie einwirkten" (SW 19; S. 204).

Mit diesen Auffassungen, die er etwa seit Beginn der 1830er Jahre und vor allem in seiner Schrift Über die Erziehung der untern Klassen der Gesellschaft (SW 19; S. 197ff.) entfaltete, behielt Diesterweg immer schärfer die äußeren Bedingungen, die sozialen Lebensumstände für die Menschenbildung im Blick, mit ihnen drang er auf eingreifende, gesellschaftliche Ungleichheit abtragende, zumindest materielle Grundsicherung herstellende Reformen. Er spürte Triebkräften der Geschichte nach, sah die Erziehung mit diesen verbunden, konstatierte Wechselwirkungen zwischen Individual- und Gesellschaftsentwicklung. Er begriff das Wesen des Menschen nicht mehr nur als Natur des Individuums, die der Erzieher zu beschauen und zu entfalten hatte, sondern in Ansätzen auch als Ausdruck gesellschaftlicher Entwicklungszustände. Dabei richteten sich seine Erwartungen auf allgemeinen moralischen, geistigen und sozialen Kulturfortschritt.

Alles in allem gewann DIESTERWEG ein im Vergleich zur bisherigen deutschen Pädagogik stärker sozial gebundenes Menschenbild. Er löste sich von der Auffassung von der, „Allmacht der Erziehung", die seiner Ansicht nach ohnehin von immer weniger Zeitgenossen geteilt werde (SW 3; S. 401f.). Im Bewusstsein der rapiden naturwissenschaftlichen, technischen, ökonomischen und sozialen Entwicklung bildete sich seine Überzeugung, dass die Erziehung innerhalb der Gesellschaft keine isolierte oder zu isolierende Tätigkeit sei. sondern in ihrer gesellschaftlichen Einbettung gesehen werden müsse. Erziehung war ihm damit mehr als nur ein Verhältnis von Erzieher und Zögling, mehr auch als das Gefüge von Erziehungseinrichtungen. Eine Fortbildung des Lebens und die Erhebung der Menschheit auf eine höhere Stufe ausschließlich oder hauptsächlich von den öffentlichen Schulen, vom Unterricht und der Schulbildung zu erwarten sei „eine große, arge Täuschung““ (SW 3; S. 51).

Gleichwohl war er doch weit davon entfernt, den unmittelbaren, großen Einfluss der Schule und sonstiger Erziehungsinstitutionen auf „die Gestaltung des äußeren Lebens“ in Abrede zu stellen. Unterricht und Erziehung seien zwar durch gesellschaftliche Einrichtungen und durch Rechtsgesetze bestimmt, aber sie wirkten auch auf diese zurück (SW 4; S. 183). Ideen von der 
Veränderung der Gesellschaft auf dem Weg der Veränderung des Individuums in „pädagogischen Provinzen" teilt er nicht er, ohne damit jedoch die große Bedeutung von Aufklärung und Erziehung zu verkennen. Es begriff Erziehung zunehmend in ihre Verflechtung mit der Gesellschaft, vertrat eine weite, soziale, auch entwicklungspsychologische Faktoren einschlieBende Erziehungsauffassung. Erziehung war für ihn „die Summe aller Einwirkungen, welche der Mensch von dem ersten Moment seines Daseins, leiblich und geistig, bewußt und unbewußt, empfangen hat“ (SW 4; 183). Könnten wir alle diese Einwirkungen, fuhr er fort, „in unsere Gewalt bringen und uns dienstbar machen, und durchschauten wir zugleich die innerste Eigentümlichkeit des zu erziehenden Wesens, so würden wir in Betreff der Erziehung alles wirken können, d. h. - allmächtig sein“.

Zwar der Meinung, man werde es so weit aus guten Gründen nicht bringen, so war er doch überzeugt, man könne sich einem solchen Zustrand zum einen durch das Fortschreiten der psychologischen Wissenschaft, zum anderen durch das Hinwirken auf eine Gesellschaft, sozialer Harmonie, weitgehender sozialer Gleichheit und gemeinschaftlicher Gesinnung nähern. Man müsse dann, um zu Tugend und Sittlichkeit, Kraft und Charakter zu erziehen, die Schule nicht mehr als „Ersatz- und Heilmittel“ zu gebrauchen suchen, sondern die Gesellschaft selbst, das Leben werde dann zu diesen Eigenschaften erziehen, der Schule dann „die leichtere, überall auch zu lösende Aufgabe des Unterweisens der Jugend“ überlassen (DSW 2, S. 542f.).

Mit den Prinzipien „Natur-“ und „Kulturgemäßheit“ hielt Diesterweg das Erziehungsdenken ausgewogen in Perspektiven, die sowohl auf das Individuum als auch auf die Gesellschaft verwiesen, damit die Verarbeitung verschiedenster Wissenschaftserträge verlangten und in den Problemen der Schule zusammenfanden. Gebunden an die Naturanlagen und ausgehend von der jeweiligen Kultur sollte der selbsttätige Mensch gleichermaßen als Individual- und als Gattungswesen höchstmögliche Entwicklungsfreiheit gewinnen. Er bezog diesen Gedanken über die scharfen sozialen Grenzen seiner Zeit hinweg auf alle Menschen. Alles in allem zielte sein Erziehungsdenken auf eine Gemeinschaft politisch mündiger und gleichberechtigter Bürger, in der diese ihre individuellen Fähigkeiten und Möglichkeiten frei entwickeln können.

\section{Werkausgaben}

Friedrich Adolph Wilhelm Diesterweg: Sämtliche Werke, hg. von Heinrich Deiters u.a., Berlin (Volk und Wissen) Bd. I-XXVII. Berlin (Ost) 1956-1990; [Forts.] hg. von Gert Geißler, Klaus Göbel, Manfred Heinemann, Horst F. Rupp und Sylvia Schütze., Berlin 1998 bis 2014: Bd. XVIII-XX, XXIII-XXIV (zitiert als SW)

Die 1956 von Erziehungswissenschaftlern in Berlin (Ost) begonnene Gesamtausgabe, die zu den umfangreichsten und anspruchvollsten pädagogischen Edition in Deutschland gehört, konnte nach 1990 fortgesetzt werden. Der 2014 erschienene Band enthält Briefe, amtliche Schreiben und Lebensdokumente aus den Jahren 1832 bis 1847

Diesterweg, Adolph: Wegweiser zur Bildung für deutsche Lehrer und andere didaktische Schriften. Ausgewählt und eingeleitet von Franz Hofmann, Berlin (Ost) 1962

Geißler, Gert./Günther, Karl.-Heinz (Hrsg.): Friedrich Adolph Wilhelm Diesterweg. Volksbildung als allgemeine Menschenbildung. 2 Bde., Berlin 1989.

Die beiden Bände bieten eine umfassende Einleitung und eine Auswahl zentraler DiesterwegTexte

Weiter Quellen

Bloth, H. G.: Adolph Diesterweg. Sein Leben und Wirken für Pädagogik und Schule, Heidelberg 1966; Geißler, G. /Rupp, H. F.: Diesterweg zwischen Forschung und Mythos. Texte und Dokument zur Forschungsgeschichte. Berlin 1996. 
Die Monographie von Bloth geht der Lebensgeschichte Diesterwegs nach und bietet eine erste Auswahl von Diesterweg-Briefen. Die Publikation von Geißler/Rupp enthält eine umfangreiche Auswahlbibliographie zur Diesterwegforschung und charakteristischer Quellentexte zur Forschungsgeschichte

Monographische Sekundärliteratur mit speziellem Themenbezug

Schröder, W.: Adolph Diesterweg. Studien zu seiner Wirkungsgeschichte in der Pädagogik des 19. und 20. Jabrhunderts, Berlin/München/Frankfurt am Main 1978; Groß, E.: Eræiehung und Gesellschaft im Werk Adolph Diesterwegs. Die Antwort der Schule auf' die soziale Frage. Weinheim 1966; Horst F. Rupp: Religion und ibre Didaktik bei Fr. A. W. Diesterweg. Ein Kapitel einer Geschichte der Religionsdidaktik im 19. Jahrbundert. Weinheim 1987.

Sammelbände

Fr.A.W. Diesterweg. Pädagogik - Lehrerbildung - Bildungspolitik. Weinheim 1990; Fichtner, B. / Menck, P. (Hg.): Pädagogik der modernen Schule. Adolph Diesterwegs Pädagogik im Zusammenhang von Gesellschaft und Schule. Weinheim/München 1992.

Ausstellungskatalog

Adolph Diesterweg. Wissen im Aufbruch. Katalog zur Ausstellung zum 200. Geburtstag. Siegen/Weinheim 1990.

In dem reich bebilderten Band geben 51 Autoren nicht nur Einblicke in Leben und Werk Diesterwegs, sondern auch in die Bildungsverhältnisse seiner Zeit. 